\title{
Cellular Communications
}

\section{Cellular Communications in the Heart}

\author{
Katerina Fountoulaki, ${ }^{1}$ Nikolaos Dagres ${ }^{2}$ and Efstathios K Iliodromitis ${ }^{2}$
}

1. Cardiothoracic Intensive Care Unit, Onassis Cardiac Surgery Centre, Athens, Greece; 2. Second University Department of Cardiology, Attikon General Hospial, University of Athens, Athens, Greece

\begin{abstract}
Heart failure is one of the leading causes of morbidity and mortality worldwide. Cardiac remodelling is first an adaptive, becoming a maladaptive, compensatory mechanism that finally causes ventricular dysfunction independently of the etiology of the initial insult. In the present article the authors describe the elements of the human heart, examining their basic functions and their inter-communication under both normal and pathological circumstances. Cardiac myocytes carry out mechanical and electrical functions of the heart and cardiac fibroblasts maintain its structural integrity. Several factors can affect fibroblast activation and under pathological stress they transdifferentiate into myofibroblasts. Endothelial cells have complex biological functions, including the control of vascular permeability, vasomotion, regulation of haemostasis, immune responses and angiogenesis. The extracellular matrix is a complex architectural network consisting of a variety of proteins. Various routes using a plethora of products and mediators contribute to the cross-talk of the myocytes with endothelial cells, extracellular matrix and cardiac fibroblasts. A better understanding of the entire mechanism of cellular communication by the established or the more recently discovered agents will certainly emerge promising new perspectives when looking at the prevention of heart failure and leading to more substantial therapeutic interventions.
\end{abstract}

\section{Keywords}

Cell communication, heart failure, cardiac remodelling, myocytes, fibroblasts

Disclosure: The authors have no conflicts of interest to declare.

Received: 24 June 2015 Accepted: 12 September 2015 Citation: Cardiac Failure Review, 2015;1(2):64-8

Correspondence: Fountoulaki Katerina, Gargittou 124-126, Gerakas 15344, Attiki, Greece. E: k_fountoulaki@hotmail.com

Heart failure (HF) is a feared endpoint for most cardiovascular diseases and is a major cause of morbidity and mortality. The worldwide prevalence of $\mathrm{HF}$ is between 2 and $3 \%$ and rises sharply at around 75 years of age, so that the prevalence in 70- to 80-year-old people is between $10-20 \%$. With a $50 \% 5$-year survival rate, $\mathrm{HF}$ is predicted to be the leading cause of all morbidity by 2020.1.2 Despite the varied etiologies, ventricular dysfunction is ultimately the result of pathologic cardiac remodelling. Cardiac remodelling is defined as a series of compensatory alterations in the size, shape, and function of the myocardium in response to cardiac injury with the aim being to restore cardiac output. However, if this process continues, chronic cardiac stress magnifies maladaptive mechanisms, including cardiac hypertrophy, fibrosis, ventricular dilatation, alteration in geometry, chronic inflammation and increased cellular apoptosis, leading to a vicious cycle of deterioration of cardiac function and worsening of HF. ${ }^{3}$

The remodelling process involves the permanent cell types of the myocardium, namely the myocytes, the fibroblasts, the endothelial cells, the smooth muscle cells and the stem cells, but also transient cell populations such as immune and circulating stem cells. ${ }^{4}$ While the cardiac myocyte (CM) has been the focus of most HF research to date, increasing evidence has implicated the cardiac fibroblast (CF) as a key pathologic determinant in cardiac remodelling in both ventricles and atria. ${ }^{5}$ Dynamic interactions among the different cardiac cell populations via mechanical, chemical and electrical means, as well as their interactions with the extracellular matrix (ECM) determine cardiac physiology and pathology. ${ }^{4,5}$ Better understanding of these cell- to-cell and cell-to-ECM communications may provide potential novel therapeutic targets for the treatment of HF. In this review, the authors aim to explore the contribution of cellular cross-talk in the cardiac remodelling process.

\section{Cellular organisation in the heart}

\section{Cardiac myocytes}

The human heart contains an estimated 2-3 billion CM cells, which constitute about $75 \%$ of the total volume of the myocardium, although only about one third of the total cell number., The major function of the $\mathrm{CM}$ is to carry out the cardiac contraction-relaxation cycle. Electrically, CM depolarise in response to signals from the sinoatrial node. Calcium is responsible for translation of the signal into muscular contraction, with calsequestrin in the sarcoplasmic reticulum being the major calcium-binding and storage protein. Mutation of this receptor can lead to a pathologic state of the myocardium, in which delayed after-depolarisation becomes prevalent.,.8

CM can act via chemical signalling by secreting various growth factors and cytokines. ${ }^{910,11,12}$ Moreover, they have been shown to exhibit a mechano-electrical feedback, in which mechanical force influences the electrical potential of the myocyte membrane. ${ }^{13}$

\section{Cardiac fibroblasts}

The majority of non-CM cells are CF. These are traditionally responsible for the maintenance of the structural integrity of the heart through regulation and turnover of the ECM. Strictly-controlled production 
and secretion of proteins, such as collagens, fibronectin, matrix metalloproteinases (MMPs), and tissue inhibitor of metalloproteinases (TIMPS), form a highly organised three-dimensional network surrounding myocytes and allow for mechanical force distribution throughout the myocardium..$^{14} \mathrm{CF}$ are cells of mesenchymal origin, but arise also from the fibrocytes, bone marrow-derived cells in the neonatal and adult heart. ${ }^{15,16,17}$ The main features of CF are the lack of a basement membrane, distinguishing them from all other permanent cardiac cells; an extensive Golgi apparatus; a relatively large endoplasmic reticulum, which underpins their role in protein synthesis and secretion; and their flat, spindle-shaped morphology with multiple filopodia originating from the main cell body. ${ }^{4}$

Under pathological stress, the resting fibroblast transdifferentiates into myofibroblast, expressing characteristics of smooth muscle cells and an enhanced capability for migration and tissue invasion. ${ }^{18,19,20}$ Moreover, myofibroblasts have been shown to originate from alternative cellular sources, including the local mesenchymal tissue, smooth muscle cells, vascular pericytes, a myeloid lineage, and fibrocytes. ${ }^{21,22}$ To date, the precise contribution of each cellular source to the myofibroblast population is poorly understood, but one hypothesis is that the transformation of differential cellular sources into myofibroblasts accounts for the manifestation of different forms of fibrosis (chronic versus acute). ${ }^{5}$ Several humoral factors can affect fibroblast activation and secretion of ECM proteins as well as their differentiation into myofibroblasts, including angiotensin II (AngII), endothelin 1 (ET-1), transforming growth factor- $\beta$ (TGF- $\beta$ ), fibroblast growth factor 2 (FGF2), and insulin-like growth factor-1 (IGF-1). 17,23,24,25 Myofibroblasts play an important role in reparative healing of the myocardium following tissue damage. ${ }^{26}$ However, imbalanced myofibroblast activity results in interstitial fibrosis, ventricular stiffening, remodelling and failure. ${ }^{27}$ An important therapeutic consideration is the degree to which cardiac fibrosis is reversible and whether myofibroblasts can undergo senescence and apoptosis or dedifferentiate back to their original cell type. ${ }^{5}$ There is evidence that two weeks after myocardial infarction about a third of myofibroblasts undergo apoptosis; nevertheless, the fate of the remaining cells remains in question. ${ }^{28}$ The current research for fibrosis is directed at understanding and inhibiting signalling pathways that regulate myofibroblast transformation.

As with $\mathrm{CM}$, fibroblasts also participate in mechano-electrical signalling which accounts for changes in the contractile function of the heart and arrhythmiogenicity in response to cardiac load alterations. ${ }^{13}$

\section{Endothelium}

The endothelial cells (EC) form the inner lining of blood vessels and as recently as the first half of the 20th century were viewed simply as barriers of blood flow. Today, endothelium has been recognised as a dynamic organ with complex biological functions, including the control of vascular permeability, the vasomotor control of coronary arteries, the regulation of haemostasis, immune responses and angiogenesis. The EC release nitric oxide, ET-1, Angll, prostaglandins, pro- and anticoagulant factors and growth factors that can affect the myocardial and vascular function. ${ }^{29}$ Furthermore, endothelium plays an important role in the regulation of heart size. ${ }^{30}$ There is evidence that the increase in myocardial vasculature is not only supportive of CM hypertrophy, but may actually induce the relevant process. ${ }^{31}$ Several studies support the notion that an increase in the capillary density is important for the development of physiological cardiac hypertrophy, whereas a reduction of the vascular bed size contributes to HF decompensation. ${ }^{32,33,34}$

\section{The Extracellular Matrix}

The cardiac ECM is a complex architectural network consisting of a variety of proteins, including fibronectin, fibrillin, periostin, more than 28 different types of collagen, glycoproteins (e.g. thrombospondins, secreted protein acidic and rich in cysteine, tenascins), proteoglycans (e.g. versican, syndecans, biglycan), and glycosaminoglycans (e.g. hyaluronan, heparan sulphate), creating strength and plasticity. ${ }^{35,36}$ Fibronectin is a multi-domain protein that interacts with proteoglycans and collagen to mediate cellular function. Periostin interacts with other components of the ECM, as well as with fibroblasts, playing a role in their differentiation. Collagen is crucial for maintaining the elasticity and integrity of the heart. Glycoproteins, proteoglycans and glycosaminoglycans are upregulated on cardiac injury and control key processes in the remodelling of myocardium, such as inflammation, fibrosis, and angiogenesis. The ECM is able to store and release a number of growth factors, chemokines and cytokines. ${ }^{4,35}$ When tissue is injured, there is an increased expression of MMPs and increased degradation of the ECM to promote healing and scar formation. Excessive degradation is blocked by endogenous TIMPs. In fact, there is a delicate balance between MMPs and TIMPs that contributes to the regulation of the cardiac remodelling proces ${ }^{37}$.

\section{Cardiac Cell-cell and Cell-ECM Communications}

\section{Cardiomyocyte to Cardiomyocyte Communication}

There are many routes of $\mathrm{CM}-\mathrm{CM}$ communication, including the secretion of autocrine factors and direct contact via gap junctions and adhesion complexes. Autocrine cross-talk is carried out by myocytesecreted factors that include leptin, hepatocyte growth factor, endothelin-1 and FGF and TGF- $\beta$ family members. ${ }^{29}$ Gap junctions allow $\mathrm{CM}-\mathrm{CM}$ communication via the exchange of ions and small solutes. In the myocardium, gap junction proteins of the connexin family have been shown to play a crucial role in determining impulse conduction and the heart morphogenesis. ${ }^{38}$ Adhesion-complex communications include intracellular signalling cascades that are triggered by cell-cell or cell-ECM engagement of specific proteins in these complexes. This type of communication may alter CM responses to growth factors leading to myocardial hypertrophy. ${ }^{29}$

Another factor released by the CM upon acute myocardial infarction is tumour necrosis factor-alpha (TNF-alpha). ${ }^{39}$ TNF-alpha has been found to be mainly released via hypoxia-inducible factor 1-alphamediated non-classical secretory pathways, involving release of vesicles containing a membrane variant of TNF. The identified vesicles were suggested to be exosomes and, when obtained from hypoxic CM, could trigger cell death in other $\mathrm{CM}^{40,41}$

\section{Cardiac Fibroblast to Myocyte Communication}

$\mathrm{CM}$ and $\mathrm{CF}$ are spatially intermingled in the myocardium with virtually every $\mathrm{CM}$ bordering one or more $\mathrm{CF}$. Bidirectional communication between CF and CM can be mediated by paracrine signals, direct cellcell interactions and indirect interaction via ECM. ${ }^{42}$

Both CF and CM secrete many different chemokines, cytokines, growth factors and other soluble agents that play a key role in cardiac physiology and pathophysiology. ${ }^{42}$ Connective tissue growth factor (CTGF), which is induced by TGF- $\beta$ and expressed in both CM and $\mathrm{CF}$, has been associated specifically with CF proliferation and ECM production in the setting of myocardial fibrosis.43,44 CTGF expression is negatively regulated by two cardiac microRNAs (miRNAs), miR-133 and miR-30. ${ }^{45}$ miRNAs are non-coding RNAs that regulate messenger 


\section{Cellular Communications}

RNA (mRNA) translation or degradation. They can be actively secreted or passively leaked from a cell to act either on an adjacent cell or on distant cells along the vasculature. ${ }^{46}$ While miR-30 is expressed in both $\mathrm{CF}$ and $\mathrm{CM}$, miR-133 is expressed specifically in CM. miR-133-knockout mice develop excessive fibrosis and HF while miR-133 knockdown also causes cardiac hypertrophy with impaired cardiac function. ${ }^{47,48} \mathrm{CF}$ also secrete interleukin 33, which cross-regulates CM in vitro, reduces pressure-overload hypertrophy and fibrosis, and improves cardiac function and survival after myocardial infarction in vivo.49,50 Interleukin 6 (IL-6) induces cell proliferation, protects cells from apoptosis, promotes ECM turnover, and causes cardiac hypertrophy. ${ }^{51}$ It is of note that interactions between $\mathrm{CM}$ and $\mathrm{CF}$ markedly promote the secretion of IL-6, as shown by increased levels in conditioned medium from $\mathrm{CM}-\mathrm{CF}$ co-cultures compared with CM or CF only cultures, indicating that communication between the two cell types is required for the secretion of some paracrine factors. ${ }^{52,53}$ Platelet-derived growth factor (PDGF) has been shown to play an important role in cardiac fibrosis and angiogenesis via its binding to protein tyrosine kinase receptors. The latter control fibroblast proliferation and migration and are also linked to ECM deposition. ${ }^{4}$ In transgenic mice, PDGF expression can be largely enhanced, leading to dilated cardiomyopathy and HF. ${ }^{54}$ IGF-1 secretion by fibroblasts also plays a key role in mediating the adaptive response of myocardium to pressure overload. ${ }^{55}$ Moreover, paracrine signals released from CF may affect the expression and function of ion channels and gap junctions in $\mathrm{CM}^{.56,5,7,5,59}$ Apart from the conventional exocytosis of paracrine factors, there is evidence that a pannexinbased mechanism exists in the myocardium. Pannexins were found to form functional channels in single membranes that can make possible paracrine intercellular communication by releasing ATP and other small molecules from the cytoplasm to the extracellular space. ${ }^{42}$

$\mathrm{CM}$ and $\mathrm{CF}$ are able to communicate electrically through connexinmediated gap junctional connections. ${ }^{4} \mathrm{CF}$ are not electrically excitable, but their membrane contains ion channels.60,61,62 Without coupling to $\mathrm{CM}, \mathrm{CF}$ operate as passive electrical insulators. ${ }^{63}$ However, when coupled to CM, they can affect action-potential characteristics and conduction velocity in $\mathrm{CM} .{ }^{42,64} \mathrm{CM}-\mathrm{CF}$ coupling has also been shown to alter intercellular calcium cycling alternans, which could play an additional role in arrhythmogenesis in fibrotic heart tissue..$^{65}$ Another mechanism for mechanical coupling between $\mathrm{CM}$ and $\mathrm{CF}$ is through adherens junctions and the cadherin-catenin complex at their core. ${ }^{42}$ Cadherins are transmembrane receptors that bind adjacent cells, link intracellularly to actin and intermediate filaments via catenins and facilitate bidirectional transmission of cytoskeletal tension between cells.66,67 Cadherin staining has been detected between co-cultured $\mathrm{CM}$ and myofibroblasts. ${ }^{68,69}$ It has been shown that TGF $\beta$-activated myofibroblasts can exert tonic contractile forces on $\mathrm{CM}$ and slow electric propagation as a result of increased mechanosensitive channel activation. ${ }^{69} \mathrm{~A}$ more recently described route of cell-cell cross talk that permits intercellular communication over longer distances is via membrane nanotubes. It has been demonstrated that organelles and cytoplasmic proteins exchange and calcium signal propagation occurred from CM to CF and vice versa through long, thin membrane nanotubular structures containing actin and microtubules. ${ }^{70,71,72}$

Cardiac Fibroblast to Endothelial Cell Communication A number of studies have demonstrated that CF can interact with EC and modify the expression of both pro- and anti-angiogenic factors. CF-secreted growth factors, including vascular endothelial growth factor (VEGF) and FGF, act on EC and stimulate angiogenesis. ${ }^{4}$
However, FC can also act in an inhibitory way. It has been shown in vitro that pigment epithelium-derived growth factor can be expressed by $\mathrm{CF}$ and can inhibit VEGF-induced tube formation. ${ }^{73}$ Moreover, the CF-secreted MMPs and TIMPS are involved in the process of angiogenesis and under certain conditions they can lead to promotion or inhibition of tube formation. ${ }^{74,75,76}$

CF and EC can also communicate by exchanging miRNAs. There is evidence that multiple miRNAs are expressed in endothelial cells and can lead to the promotion or inhibition of angiogenesis. ${ }^{77.78}$

\section{Cardiac Myocyte to Endothelial Cell Communication}

In the heart, EC outnumber CM at nearly a 3:1 ratio with virtually every $\mathrm{CM}$ bordering one or more capillary. ${ }^{79} \mathrm{CM}$ paracrine signalling plays a key role in the dynamic regulation of the vascular tone but may also affect long term growth and development of coronary arterial, venous and lymphatic trees. ${ }^{29}$ Among the multiple paracrine signals the most important are VEGFs. When there is either an overexpression or a deficit in VEGF, the ultimate result is cardiac dysfunction. ${ }^{80}$ It is of note that although CM represent less than a third of the total cell number in the heart, cardiomyocyte-specific deletion of the VEGF-A gene results in a decrease in the entire VEGF mRNA synthesis to less than $15 \%$ of normal, emphasising the role of $\mathrm{CM}$ as the main source of this growth factor in the myocardium. ${ }^{29}$ When mice were engineered with cardiac myocyte-specific deletion of VEGF, the result was thinned ventricular walls, decreased contractile function and lack of neural stimulation. ${ }^{81}$ VEGF is considerably increased in the ischemic myocardium, whereas the vasculature in ischemic myocardium is more sensitive to VEGFinduced vasodilatation. .2,83 $^{2}$

CM-EC communication is very important for the vascular adaptations that occur during cardiac hypertrophy. Myocardial hypertrophy induced by expression of Akt1 is a clinical setting that illustrates the importance of the balance between cardiac and vascular growth. Transgenic overexpression of activated Akt1 in CM resulted in a varied spectrum of phenotypes from myocardial hypertrophy with preserved systolic function to ventricular dilatation and failure. ${ }^{84}$ In a tetracycline-inducible cardiac myocyte-specific Akt1 transgenic mouse model, short-term (two weeks) induction of Akt1 expression resulted in physiological hypertrophy that was accompanied by analogous myocardial angiogenesis. ${ }^{85}$ However, Akt1 activation for longer periods of time resulted in a disproportionate increase in cardiac mass compared with the extent of angiogenesis and development of HF, presumably on the basis of an inadequate blood supply to cover the requirements of the hypertrophic myocardium. ${ }^{85} \mathrm{CM}$ and $\mathrm{EC}$ are able to communicate electromechanically through gap junction proteins, such as $\mathrm{C} \times 43 .{ }^{86}$ and it has been demonstrated that VEGF can affect $\mathrm{C} \times 43$ expression in $\mathrm{CM}$, suggesting another VEGF-dependent pathway as a mode of interaction between myocardium and vasculature. ${ }^{80}$

\section{Cell to Matrix Communication}

The principal mediators of molecular dialogue between a cell and its extracellular matrix environment are integrins. ${ }^{87}$ They are heterodimeric cell-surface molecules that mediate signalling from the extracellular space into the cell through integrin-associated signalling and adaptor molecules such as focal adhesion kinase, integrin-linked kinase particularly interesting new cysteine-histidine rich protein and non-catalytic (region of) tyrosine kinase adaptor protein-2. ${ }^{.7}$ Via these molecules, integrin signalling interacts with receptor tyrosine kinases signalling to regulate survival, proliferation and cell shape as well as 
polarity, adhesion, migration and differentiation. In the myocardium and blood vessels, the function and regulation of these molecules can be partially disturbed, leading to cardiovascular diseases such as cardiac hypertrophy and atherosclerosis. Integrin-mediated cell adhesion can be modulated by membrane-associated proteins such as the ADAMs (a desintegrin and metalloprotease). ADAMs can alter expression and function of growth factor receptors and, as a result, will then affect several biological processes, including angiogenesis and cardiac hypertrophy. ${ }^{29,88}$

\section{Conclusions and Therapeutic Perspectives}

Each cell type in the myocardium interacts with another in various ways and has its own unique changes to gene and protein expression, protein secretion and response to signals from other cells (Figure 1). It has become clear that the myofibroblast is a significantly more complex cell type that was initially appreciated. Moreover, the ECM has emerged as a dynamic component in both the normal and diseased heart. Despite recent progress, our understanding of many of these cell-cell and cell-ECM interactions remains unclear.

Cytokine antagonists, particularly those targeting $T N F \alpha$, did not provide any apparent clinical benefit in HF patients, despite the promising preclinical data, but targeting the role of inflammatory cytokine signalling in HF has provided fruitful lessons for future drug development. Moreover, it would be highly beneficial to identify pharmacologic agents that might inhibit myofibroblast formation in chronic disease states while sparing their protective role in physiological wound healing. One particularly important aspect of
Figure 1: Schematic Diagram Illustrating Multiple Mechanisms of Cardiac Intercellular Communication

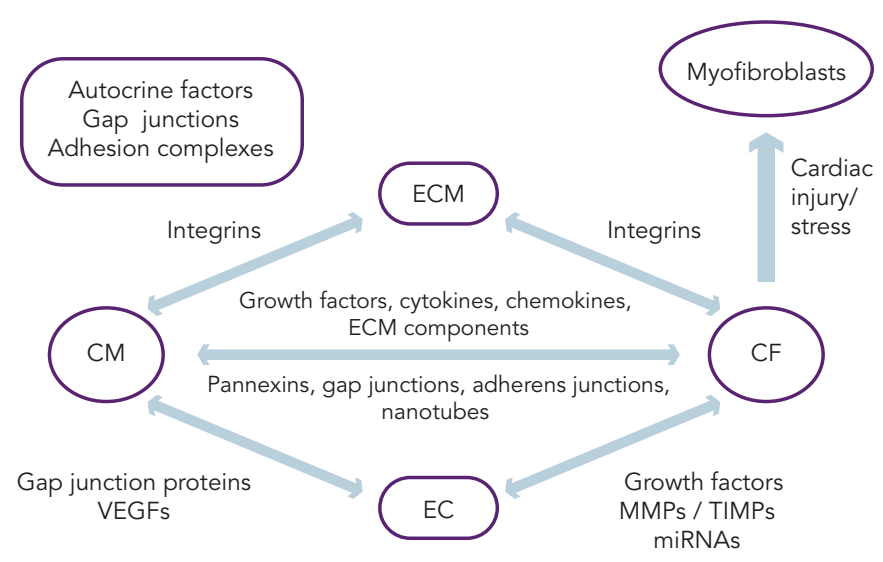

$C M=$ cardiac myocytes; $C F=$ cardiac fibroblasts; $E C=$ endothelial cells; $E C M=$ extracellular matrix; MMPS = matrix metalloproteinases; TIMPS = Tissue inhibitor of metalloproteinase; VEGFS = vascular endothelial growth factors

cell-cell cross-talk in the heart is the CM-EC interactions. Once our understanding of how changes in endothelial cell mass affect cardiac hypertrophy improves, we may gain more insight into the possibility of stimulating angiogenesis and vessel growth in the injured myocardium.

Further research into the dynamic intercellular communication is crucial for the development of novel therapeutic strategies that target directly HF disease progression.
1. Dickstein K, Cohen-Solal A, Filippatos G, et al. ESC Committee for Practice Guidelines (CPG). ESC Guidelines for the diagnosis and treatment of acute and chronic heart failure 2008: the Task Force for the Diagnosis and Treatment of Acute and Chronic Heart Failure 2008 of the European Society of Cardiology. Developed in collaboration with the Heart Failure Association of the ESC (HFA) and endorsed by the European Society of Intensive Care Medicine (ESICM). Eur Heart J 2008:29:2388-442.

2. Roger VL, Go AS, Lloyd-Jones DM, et al. American Heart Association Statistics Committee and Stroke Statistics Subcommittee. Heart disease and stroke statistics-2011 update: a report from the American Heart Association. circulation 2011;123:e18-e209.

3. Hill JA, OIson EN. Cardiac plasticity. N Eng/ J Med 2008;27;358(13):1370-80.

4. Howard CM, Baudino TA. Dynamic cell-cell and cell-ECM interactions in the heart. J Mol Cell Cardiol 2014;70:19-26.

5. Davis J, Molkentin JD. Myofibroblasts: trust your heart and let fate decide. J Mol Cell Cardiol 2014;70:9-18.

6. Banerjee I, Fuseler JW, Price RL, et al. Determination of cell types and numbers during cardiac development in the neonatal and adult rat and mouse. Am J Physiol Heart Circ Physiol 2007:293:H1883-91.

7. Nag AC. Study of non-muscle cells of the adult mammalian heart: a fine structural analysis and distribution. Cytobios 1980:28:41-61.

8. Fearnley CJ, Roderick HL, Bootman MD. Calcium signalling in cardiac myocytes. Cold Spring Harb Perspect Biol 2011;3:1-21.

Ballard-Croft C, White DJ, Maass DL, et al. Role of p38 mitogen-activated protein kinase in cardiac myocyte secretion of the inflammatory cytokine TNF-alpha. Am J Physiol Heart Circ Physiol 2001:280:H1970-81.

10. Bryant $D$, Becker $L$, Richardson J, et al. Cardiac failure in transgenic mice with myocardial expression of tumour necrosis factor-alpha. Circulation 1998 14:97:1375-81.

11. Bowers SL, Baudino TA. Cardiac myocyte-fibroblas interactions and the coronary vasculature. J Cardiovasc Trans Res 2012;5:783-93.

12. Schulz R, Nava E, Moncada S. Induction and potential biological relevance of a $\mathrm{Ca}(2+)$-independent nitric oxide synthase in the myocardium. Br J Pharmacol 1992;105:575-80.

13. Kamkin A, Kiseleva I, Lozinsky I, Scholz H. Electrical interaction of mechanosensitive fibroblasts and myocytes in the heart. Basic Res Cardiol 2005; 100:337-45.

14. Martin ML, Blaxall BC. Cardiac intercellular communication: are myocytes and fibroblasts fair-weather friends? J Cardiovasc Transl Res 2012;5:768-82.

15. Katz TC, Singh MK, Degenhardt K, et al. Distinct compartments of the proepicardial organ give rise to coronary vascular endothelial cells. Dev Cell 2012;22:639-50.
6. Kanekar S, Hirozanne T, Terracio L, Borg TK. Cardiac fibroblasts form and function. Cardiovasc Pathol 1998:7:127-33. 7. Souders CA, Bowers SL, Baudino TA. Cardiac fibroblast: the renaissance cell. Circ Res 2009;105:1164-76.

18. Tomasek JJ, Gabbiani G, Hinz B, et al. Myofibroblasts and mechano-regulation of connective tissue remodelling. Nat Rev Mol Cell Biol 2002;3:349-63.

19. Leeb SN, Vogl D, Falk W, et al. Regulation of migration of human colonic myofibroblasts. Growth Factors 2002:20:81-91.

20. Suganuma H, Sato A, Tamura R, Chida K. Enhanced migration of fibroblasts derived from lungs with fibrotic lesions. Thorax 1995;50:984-9.

21. Zeisberg EM, Kalluri R. Origins of cardiac fibroblasts. Circ Res 2010;107:1304-12

22. Crawford JR, Haudek SB, Cieslik KA, et al. Origin of developmental precursors dictates the pathophysiologic role of cardiac fibroblasts. I Cardiovasc Transl Res 2012;5:749-59.

23. Manabe I, Shindo T, Nagai R. Gene expression in fibroblasts and fibrosis: involvement in cardiac hypertrophy. Circ Res 2002:91:1103-13.

24. Bujak M, Frangogiannis NG. The role of TGF-beta signalling in myocardial infarction and cardiac remodeling. Cardiovasc Res 2007:74:184-95.

25. Bouzegrhane $F$, Thibault $G$. Is angiotensin II a proliferative factor of cardiac fibroblasts? Cardiovasc Res 2002;53:304-12.

26. Calderone A, Bel-Hadj S, Drapeau J, et al. Scar myofibroblasts of the infarcted rat heart express natriuretic peptides. of the infarcted rat heart express

27. Porter KE, Turner NA. Cardiac fibroblasts: at the heart of myocardial remodelling. Pharmacol Ther 2009:123:255-78.

28. Takemura G, Ohno M, Hayakawa $Y$, et al. Role of apoptosis in the disappearance of infiltrated and proliferated interstitia cells after myocardial infarction. Circ Res 1998:82:1130-8.

29. Tirziu D, Giordano FJ, Simons M. Cell communications in the heart. Circulation 2010;122:928-37.

30. Tirziu D, Simons M. Endothelium as master regulator of organ development and growth. Vascul Pharmacol 2009;50:1-7.

31. Tomanek RJ, Busch TL. Coordinated capillary and myocardia growth in response to thyroxine treatment. Anat Rec 1998;251:44-9.

32. Izumiya Y, Shiojima I, Sato K, et al. Vascular endothelial growth factor blockade promotes the transition from compensatory cardiac hypertrophy to failure in response to pressure overload. Hypertension 2006;47:887-93.

33. Sano M, Minamino T, Toko H, et al. p53-induced inhibition of Hif-1 causes cardiac dysfunction during pressure overload. Nature. 2007;446:444-8.

34. Dorn GW 2nd. Myocardial angiogenesis: its absence makes the growing heart founder. Cell Metab 2007;5:326-7.

35. Lockhart M, Wirrig E, Phelps A, Wessels A. Extracellular matrix and heart development. Birth Defects Res A Clin Mol Teratol
2011;91:535-50.

36. Rienks M, Papageorgiou AP, Frangogiannis NG, Heymans S. Myocardial extracellular matrix, an ever-changing and diverse entity. Circ Res 2014;114:872-88.

37. Raffetto JD, Khalil RA. Matrix metalloproteinases and their inhibitors in vascular remodelling and vascular disease. Biochem Pharmacol 2008;75:346-59

38. Lo CW. Role of gap junctions in cardiac conduction and development: insights from the connexin knockout mice. Circ Res 2000;87:346-8.

39. Tracey KJ, Cerami A. Tumor necrosis factor, other cytokines and disease. Annu Rev Cell Biol 1993;9:317-43.

40. Yu X, Deng L, Wang $D$, et al. Mechanism of TNF- $\alpha$ autocrine effects in hypoxic cardiomyocytes: initiated by hypoxia inducible factor $1 \alpha$, presented by exosomes. J Mol Cell Cardio 2012;53:848-57.

41. Sluijter JP, Verhage V, Deddens JC, et al. Microvesicles and exosomes for intracardiac communication. Cardiovasc Res 2014;102:302-11.

42. Zhang P, Su J, Mende U. Cross-talk between cardiac myocytes and fibroblasts: from multiscale investigative approaches to mechanisms and functional consequences. Am J Physiol Heart Circ Physiol 2012;303:H1385-96.

43. Chen MM, Lam A, Abraham JA, et al. CTGF expression is induced by TGF-beta in cardiac fibroblasts and cardiac myocytes: a potential role in heart fibrosis. I Mol Cell Cardiol 2000:32:1805-19.

44. Zhang J, Chang L, Chen C, et al. Rad GTPase inhibits cardiac fibrosis through connective tissue growth factor. Cardiovasc Res 2011:91:90-8.

45. Duisters RF, Tijsen AJ, Schroen B, et al. miR-133 and miR-30 regulate connective tissue growth factor: implications for a role of microRNAs in myocardial matrix remodeling. Circ Res 2009;104:170-8.

46. Doebele C, Bonauer A, Fischer A, et al. Members of the microRNA-17-92 cluster exhibit acell-intrinsic antiangiogenic function in endothelial cells. Blood 2010;115:4944-50.

7. Carè A, Catalucci D, Felicetti F, et al. MicroRNA-133 controls cardiac hypertrophy. Nat Med 2007:13:613-8.

48. van Rooij E, Olson EN. Searching for miR-acles in cardiac fibrosis. Circ Res 2009;104:138-40.

49. Sanada S, Hakuno D, Higgins L, et al. LL-33 and ST2 comprise a critical biomechanically induced and cardioprotective signalling system. J Clin Invest 2007;117:1538-49.

50. Seki K, Sanada S, Kudinova AY, et al. Interleukin-33 prevents apoptosis and improves survival after experimental myocardial infarction through ST2 signaling. Circ Heart Fall 2009;2:684-91

51. Ottaviano FG, Yee KO. Communication signals between cardiac fibroblasts and cardiac myocytes. I Cardiovasc Pharmacol 2011;57:513-21. 
52. Bowers SL, Borg TK, Baudino TA. The dynamics of fibroblastmyocyte-capillary interactions in the heart. Ann N Y Acad SCl 2010;1188:143-52.

53. Sarkar S, Vellaichamy E, Young D, Sen S. Influence of cytokines and growth factors in ANG II-mediated collagen upregulation by fibroblasts in rats: role of myocytes. Am J Physiol Heart Circ Physiol 2004;287:H107-17.

54. Pontén A, Li X, Thorén P, et al. Transgenic overexpression of platelet-derived growth factor- $\mathrm{C}$ in the mouse heart induces cardiac fibrosis, hypertrophy, and dilated cardiomyopathy. Am J Pathol 2003;163:673-82.

55. Takeda N, Manabe I, Uchino Y, et al. Cardiac fibroblasts are essential for the adaptive response of the murine heart to pressure overload. I Clin Invest 2010;120:254-65.

56. Doble BW, Chen Y, BOSC DG, et al. Fibroblast growth factor-2 decreases metabolic coupling and stimulates phosphorylation as well as masking of connex

57. Goette A, Lendeckel U. Electrophysiological effects of angiotensin II. Part I: signal transduction and basic electrophysiological mechanisms. Europace 2008;10:238-41.

5. Merle PL, Feige JJ, Verdetti J. Basic fibroblast growth factor activates calcium channels in neonatal rat cardiomyocytes. biol Chem 1995;270:17361-7.

59. Teos LY, Zhao A, Alvin Z, et al. Basal and IGF-I-dependent regulation of potassium channels by MAP kinases and PI3kinase during eccentric cardiac hypertrophy. Am I Physiol Heart Circ Physiol 2008:295:H1834-45.

60. Chilton L, Ohya S, Freed D, et al. K+ currents regulate the Chesting esting membrane potential, proliferation, and contractile Am I Physiol Heart Circ Physiol 2005:288: H2931-9.

61. Li GR, Sun HY, Chen JB, et al. Characterization of multiple ion channels in cultured human cardiac fibroblasts. PLOS One 2009; 4:e7307.

62. Vasquez C, Benamer N, Morley GE. The cardiac fibroblast: functional and electrophysiological considerations in health and diseased hearts. I Cardiovasc Pharmacol 2011;57:380-8.

63. Xie Y, Garfinkel A, Camelliti P, Kohl P, et al. Effects of fibroblast-myocyte coupling on cardiac conduction and vulnerability to reentry: A computational study. Heart Rhythm
2009;6:1641-9

64. Kohl P, camelliti P, Burton FL, Smith GL. Electrical coupling of fibroblasts and myocytes: relevance for cardiac propagation. J Electrocardiol 2005;38:45-50.

65. Xie Y, Garfinkel A, Weiss JN, Qu Z. Cardiac alternans induced by fibroblast-myocyte coupling: mechanistic insights from computational models. Am J Physiol Heart Circ Physiol 2009;297:H775-84.

66. Harris TJ, Tepass U. Adherens junctions: from molecules to morphogenesis. Nat Rev Mol Cell Biol 2010;11:502-14.

67. McCain ML, Parker KK. Mechanotransduction: the role of mechanical stress, myocyte shape, and cytoskeletal architecture on cardiac function. Pflugers Arch 2011;462(1):89-104

68. Pedrotty DM, Klinger RY, Badie N, et al. Structural coupling of cardiomyocytes and noncardiomyocytes: quantitative comparisons using a novel micropatterned cell pair assay. Am J Physiol Heart Circ Physiol 2008;295:H390-400.

69. Thompson SA, Copeland CR, Reich DH, Tung L. Mechanical coupling between myofibroblasts and cardiomyocytes slows electric conduction in fibrotic cell monolayers. Circulation 2011;123:2083-93.

70. He K, Shi X, Zhang X, et al. Long-distance intercellular connectivity between cardiomyocytes and cardiofibroblasts mediated by membrane nanotubes. Cardiovasc Res 2011;92:39-47.

71. Davis DM, Sowinski S. Membrane nanotubes: dynamic long. distance connections between animal cells. Nat Rev Mol Cell distance connections $2008 ; 9: 431-6$.

72. Wang $\mathrm{X}$, Gerdes $\mathrm{HH}$. Long-distance electrical coupling via tunneling nanotubes. Biochim Biophys Acta 2012;1818:2082-6.

73. Rychli $K$, Kaun $C$, Hohensinner PJ, et al. The anti-angiogenic factor PEDF is present in the human heart and is regulated by anoxia in cardiac myocytes and fibroblasts. 1 Cell Mol Med 2010;14:198-205.

74. Powell DW, Mifflin RC, Valentich JD, et al. Myofibroblasts, I. Paracrine cells important in health and disease. Am J Physiol 1999:277:C1-9.

75. Lambert $E$, Dassé $E$, Haye B, Petitfrère E. TIMPS as multifacial proteins. Crit Rev Oncol Hematol 2004:49:187-98.

76. Liu H, Chen B, Lilly B. Fibroblasts potentiate blood vessel formation partially through secreted factor TIMP-1. Angiogenesis 2008;11:223-34.

77. Kukreja RC, Yin C, Salloum FN. MicroRNAs: new players in cardiac injury and protection. Mol Pharmacol 2011;80:558-64.

78. Bonauer A, Carmona G, Iwasaki M, et al. MicroRNA-92a controls angiogenesis and functional recovery of ischemic tissues in mice. Science 2009 26;324:1710-3.

79. Brutsaert DL. Cardiac endothelial-myocardial signalling: its role in cardiac growth, contractile performance, and rhythmicity. Physiol Rev 2003;83:59-115.

80. Narmoneva DA, Vukmirovic R, Davis ME, et al. Endothelial cells promote cardiac myocyte survival and spatial reorganization: implications for cardiac regeneration. Circulation 2004;110(8):962-8.

81. Giordano FJ, Gerber HP, Williams SP, et al. A cardiac myocyte vascular endothelial growth factor paracrin pathway is required to maintain cardiac function. Proc Natl Acad Sci U S A 2001;98:5780-5.

82. Li J, Brown LF, Hibberd MG, Grossman JD, et al. VEGF, flk-1, and flt-1 expression in a rat myocardial infarction model of angiogenesis. Am I Physiol 1996:270: H1803-11.

83. Sellke FW, Wang SY, Stamler A, et al. Enhanced microvascular relaxations to VEGF and bFGF in chronically ischemic porcine myocardium. Am J Physiol 1996;271:H713-20.

84. Matsui T, Li L, Wu JC, et al. Phenotypic spectrum caused by transgenic overexpression of activated Akt in the heart. J Biol Chem 2002:277:22896-901.

85. Shiojima I, Sato K, Izumiya Y, et al. Disruption of coordinated cardiac hypertrophy and angiogenesis contributes to the cardiac hypertrophy and angiogenesis contributes to the

86. Givvimani S, Qipshidze N, Tyagi N, et al. Synergism between arrhythmia and hyperhomocysteinemia in structural heart

87. Lat H, Verma SK, Foster DM et at. Incerins a Lal H, Verma SK, Foster DM, et al. Integrins and proximal
signaling mechanisms in cardiovascular disease. Front Biosci (Landmark Ed) 2009:14:2307-34.

88. Asakura M, Kitakaze M, Takashima S, et al. Cardiac hypertrophy is inhibited by antagonism of ADAM12 processing of HB-EGF: metalloproteinase inhibitors as new therapy. Nat Med 2002:8:35-40. 